\title{
Nonequilibrium drift-diffusion model for organic semiconductor devices
}

\author{
Nikolaos Felekidis, ${ }^{1}$ Armantas Melianas, ${ }^{2}$ and Martijn Kemerink ${ }^{1}$ \\ ${ }^{1}$ Complex Materials and Devices, Department of Physics, Chemistry and Biology, Linköping University, 58183 Linköping, Sweden \\ ${ }^{2}$ Biomolecular and Organic Electronics, Department of Physics, Chemistry and Biology, Linköping University, 58183 Linköping, Sweden
}

(Received 18 May 2016; revised manuscript received 24 June 2016; published 27 July 2016)

\begin{abstract}
Two prevailing formalisms are currently used to model charge transport in organic semiconductor devices. Drift-diffusion calculations, on the one hand, are time effective but assume local thermodynamic equilibrium, which is not always realistic. Kinetic Monte Carlo models, on the other hand, do not require this assumption but are computationally expensive. Here, we present a nonequilibrium drift-diffusion model that bridges this gap by fusing the established multiple trap and release formalism with the drift-diffusion transport equation. For a prototypical photovoltaic system the model is shown to quantitatively describe, with a single set of parameters, experiments probing (1) temperature-dependent steady-state charge transport—space-charge limited currents, and (2) time-resolved charge transport and relaxation of nonequilibrated photocreated charges. Moreover, the outputs of the developed kinetic drift-diffusion model are an order of magnitude, or more, faster to compute and in good agreement with kinetic Monte Carlo calculations.
\end{abstract}

DOI: 10.1103/PhysRevB.94.035205

\section{INTRODUCTION}

As is characteristic of science, the field of organic electronics has benefited tremendously from quantitative modeling of material properties and device performance. Roughly speaking, two formalisms can cope with the length scale set by the typical active layer thickness in organic thin film devices of $\sim 100 \mathrm{~nm}$ : drift-diffusion (DD) and kinetic Monte Carlo (MC). For realistic devices that, e.g., include contacts, neither of the two models allows for analytical solutions and one has to rely on numerical techniques. Kinetic MC has been extremely successful in describing the effects of static energetic disorder on the charge transport in disordered organic semiconductors [1-5]. As MC typically puts high demands on computation power, a common and successful approach has been to incorporate the molecular detail into the simpler DD formalism by means of a mobility functional [6-9]. A range of parameterizations of the temperature and electric field dependence of the mobility stemming from numerical simulations that are typically performed on regular lattices has been reported [1-3,10,11]. In addition, various analytical expressions on basis of, typically, variable range hopping models exist [3,12-14]. An alternative approach is the inclusion of (a distribution of) trap states residing below the band considered in DD [15-17]. These approaches allow capture, in a DD model, of a significant part of the phenomena brought about by the conduction mechanism of charge carrier hopping in an energetically disordered density of states (DOS).

The DD model is inherently based on the (assumed) presence of well-defined (quasi-)Fermi energies, and hence on the existence of (local) thermodynamic equilibrium through the underlying transport equation $j=q n \mu \nabla E_{F}$. Here, $j$ and $n$ are the current and charge density, $\mu$ is the mobility, and $E_{F}$ is the quasi-Fermi level. This implies that phenomena related to the slow process of charges seeking their way to thermodynamic equilibrium in the DOS cannot be accounted for. It is well known that such long-range relaxation processes can be significantly slow in a strongly energy-dependent DOS, such as an exponential or a Gaussian $[1,18-20]$. In recent papers, we have shown that in typical organic solar cells, these relaxation processes may take longer than the extraction of photocreated charge carriers from an operating device $[21,22]$. This observation makes widespread use of equilibrium charge carrier mobilities and quasi-Fermi levels of questionable value when interpreting the characteristics of organic solar cells and related devices such as photodetectors; the impact of associated errors is, however, largely unknown.

In view of the above, we think there is a need for a formalism that combines the ability of kinetic MC models to naturally deal with nonequilibrium phenomena with the computational ease of DD modeling. Here, we develop such a hybrid kinetic drift-diffusion (kDD) device model. The model produces a consistent description of measured space-charge limited currents and ultrafast charge extraction experiments. Moreover, it is validated by direct comparison with transient and steady-state kinetic MC calculations.

\section{THEORY}

The model is based on the multiple trapping and release (MTR) formalism that was originally used to describe relaxation phenomena in amorphous inorganic semiconductors having a deep low-energy tail in their DOS [19,23-25]. Recently, Germs et al. adapted the MTR formalism to describe impedance spectroscopy performed on hole-only diodes based on organic semiconductors with a Gaussian DOS [26]. We have extended their single-site model to deal with situations in which the finite thickness of the device cannot be ignored, e.g., space-charge limited diodes and time-resolved charge extraction experiments. In the following, we outline the basic equations of our one-dimensional (1D) transport model.

\section{A. Basic equations}

In the MTR formalism, a mobility edge is defined at an energy $E_{c}$ above which the charge carriers are mobile. Below the mobility edge, there is a distribution of localized states (in 


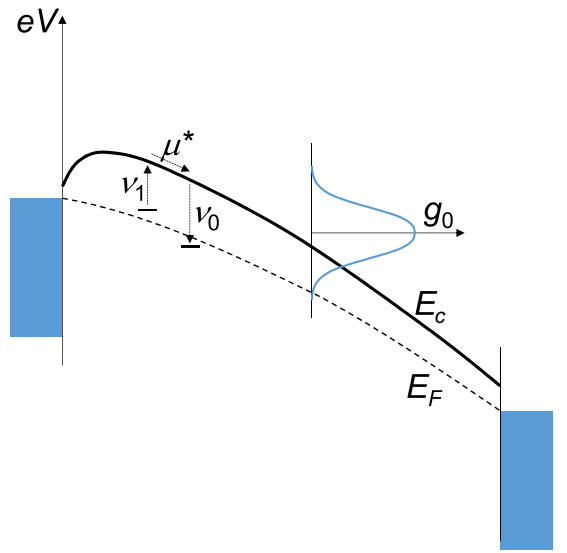

FIG. 1. Schematic band diagram under near-equilibrium conditions, indicating the electron mobility edge $E_{c}$ and the quasi-Fermi level $E_{F}$ in the disordered semiconductor that is sandwiched between two metallic contacts (blue areas). The arrows indicate a single release-transport-trapping cycle characterized by rates $v_{1,0}$ and free mobility $\mu^{*}$. The blue curve indicates the Gaussian DOS $g_{0}(\mathrm{E})$.

our case, a Gaussian) (see also Fig. 1):

$$
g_{0}(E)=\frac{\beta N_{i}}{\sqrt{2 \pi} \sigma_{\mathrm{DOS}}} \exp \left(-\frac{\left(E-E_{H / L}-q V(z)\right)^{2}}{2 \sigma_{\mathrm{DOS}}^{2}}\right)
$$

In Eq. (1), $\beta N_{i}$ is the total site density, $\sigma_{\text {DOS }}$ is the width of the Gaussian, $V(z)$ is the electrostatic potential at position $z$ in the device, and $E_{H / L}$ is the highest/lowest occupied molecular orbital energy. The factor $\beta$ is on the order of 2 and accounts for the fact that in the MTR formalism, only the fraction of the DOS below $E_{c}$, corresponding to a site density $N_{i}$, is considered. In fact, $\beta$ is easily calculated from $\beta=\int_{-\infty}^{\infty} g_{0}(E) / \int_{-\infty}^{E_{C}} g_{0}(E)$.

Transport takes place by charges that have been thermally excited from the localized states to the mobility edge at a rate

$$
v_{1}=v_{0} \exp \left(-\frac{E_{c}-E_{\text {trap }}}{k_{B} T}\right)
$$

where $E_{\text {trap }}$ is the energy of the trap site from which detrapping takes place and $v_{0}$ is the attempt frequency. Trapping into an empty site occurs at the rate $v_{0}$. Between the detrapping and the retrapping events, the charge carrier moves with a free mobility, given by the following [2,26]:

$$
\mu^{*}=v_{0} a_{\mathrm{NN}}^{2} / \sigma_{\mathrm{DOS}}
$$

The nearest-neighbor distance $a_{\mathrm{NN}}$ is related to the total DOS as $a_{\mathrm{NN}}=\left(\beta N_{i}\right)^{-1 / 3}$. The parameter $\beta$ enables comparison between the present formalism and the MC calculations in which the full DOS is commonly used at a constant $a_{\mathrm{NN}}$. The free mobility is related to the near-equilibrium mobility at low field and low charge carrier concentration $\mu_{0}[2,26,27]$

$$
\mu_{0}=\mu^{*} \exp \left(-\chi \hat{\sigma}^{2}\right)
$$

with $\hat{\sigma}=\sigma / k_{B} T$ and $\chi \approx 0.44$.

In order to capture the enhancement of the mobility by an electric field $F$, we make the following ansatz for the position of the mobility edge:

$$
E_{c}=E_{c, 0}\left(1+\alpha\left(\frac{q a_{\mathrm{NN}} F}{\sigma}\right)^{2}\right)^{\frac{1}{2}}
$$

In Eq. (5), $\alpha$ is a scaling constant of order of unity. In the comparison with kinetic MC simulations discussed below, we find good correspondence between MC and $\mathrm{kDD}$ for $\alpha=2.2$. This value is used for all calculations shown in this paper. $E_{c, 0}$ is the zero-field mobility edge, for which we use the critical energy, as found by Cottaar et al. [27]. For Miller-Abrahams hopping on a simple cubic lattice, $E_{c, 0}=-0.491 \sigma$.

The particular form of the ansatz Eq. (5) is inspired by the fact that the lowering of the final site energy of the characteristic hop must be linear in $F$ at high fields and symmetric at low fields to avoid unphysical discontinuities at $F=0$. The scaling of the field energy term $q a_{\mathrm{NN}} F$ by the disorder strength $\sigma_{\mathrm{DOS}}$ is in the spirit of Eq. (3), in which the free mobility is obtained by dividing the diffusion constant $v_{0} a_{\mathrm{NN}}^{2}$ by $\sigma_{\mathrm{DOS}}$, not by $k_{B} T$. We verified that temperature-dependent scaling factors (such as $k_{B} T$ ) in Eq. (5) failed to reproduce both the kinetic MC simulations and the experiments discussed below, whereas the scaling introduced in Eq. (5) successfully reproduces both. Finally, it should be kept in mind that while the lowering of the mobility edge with field is temperature independent, its effect on the mobility increases strongly at lower $T$ due to the thermal activation of the detrapping process described in Eq. (2).

During its time $t_{\text {free }}$ at the transport energy (above the mobility edge), the charge carrier travels the following distance:

$$
\Delta z=\mu^{*} F t_{\text {free }} \pm \sqrt{2 D^{*} t_{\text {free }}}
$$

The first term accounts for drift motion and gets a minus sign for electron motion. The second term accounts for $1 \mathrm{D}$ diffusion. Note that $\Delta z$ is a continuous variable and not restricted to discrete values corresponding to a lattice. The direction of the diffusion is randomly chosen for each event. The diffusion constant is related to the mobility as $D^{*}=\mu^{*} k_{B} T$. Even though the use of the Einstein relation to connect $\mu^{*}$ and $D^{*}$ does not imply that the same relation holds for the mobility $\mu$ and diffusion constant $D$ of the system as a whole, its use is not evident upfront, because it implicitly assumes thermodynamic equilibrium in the transport level. We have tested the alternative expression $D^{*}=\mu^{*} \sigma_{\mathrm{DOS}}=v_{0} a_{\mathrm{NN}}^{2}$, which was inspired by Eq. (3) and the ansatz in Eq. (5). This failed to reproduce both the kinetic MC simulations and the experiments discussed below. In particular, it led to an overestimation of the diffusion at lower $T$, giving rise to an overly weak temperature dependence of space-charge limited currents in devices. In the present formalism, we therefore interpret the transport level as a phenomenological, disorder-free band sitting at the mobility edge; apparently, complications associated with the Gaussian disorder can to a large degree be mapped on trapping in the localized states below it. In the limit of equilibrium transport, this interpretation is (implicit) common practice in modeling of organic field-effect transistors (see, e.g., Ref. [28] and references therein). Finally, in Ref. [27], the zero-field mobility edge $E_{c, 0}$ was shown to have the physical meaning of being 
the highest energy of sites participating in the critical bonds of the percolating network formed on a regular lattice with nearest-neighbor hopping.

In the spirit of DD calculations, interactions between charges are treated through the mean carrier distributions. The Coulomb interaction can thus be treated through the 1D Poisson equation

$$
\frac{d^{2} V}{d z^{2}}=-\frac{\rho(z)}{\varepsilon_{0} \varepsilon_{r}}
$$

where $V$ is the electrostatic potential, $\rho$ is the total charge density, and $\varepsilon_{0} \varepsilon_{r}$ is the dielectric constant of the semiconductor. Metallic contacts at $z=0$ and $z=L$ set the boundary conditions required to determine $V$. The image force interaction with the contacts is included through the Simmons approximation [29]:

$$
V_{\operatorname{im}}(z)=-\frac{1.15 q^{2} L \ln 2}{8 \pi \varepsilon_{0} \varepsilon_{r}} \frac{1}{z(L-z)}
$$

A practicality associated with the divergences of this equation is discussed in the Appendix.

Recombination of charges of one polarity with charges of the other polarity consists of two contributions. When free, a hole can recombine with free or trapped electrons with the following rate $[9,30]$ :

$$
v_{h, f}=\frac{q}{\varepsilon_{0} \varepsilon_{r}} \mu_{h}^{*}\left(n_{e, t}+n_{e, f}\right)
$$

The subscripts $e$ and $h$ refer to electrons and holes, and $t$ and $f$ refer to trapped and free, respectively. The rate for recombination of a trapped hole with free electrons is as follows:

$$
v_{h, t}=\frac{q}{\varepsilon_{0} \varepsilon_{r}} \mu_{e}^{*} n_{e, f}
$$

Similar expressions for electron recombination are easily written. It was checked that for steady-state transport under near-equilibrium conditions, the combined effect of Eqs. (9a) and 9(b) corresponds to the conventional Langevin recombination rate $R=v_{r, L} n p$, with $n$ and $p$ indicating the total electron and hole densities and $v_{r, L}=\frac{q}{\varepsilon_{0} \varepsilon_{r}}\left(\mu_{0, e}+\mu_{0, h}\right)$. When needed, sub-Langevin recombination is readily introduced by adding a Langevin reduction prefactor smaller than unity in Eqs. (9a) and 9(b); in the following, this option is not further pursued.

\section{B. Numerical implementation}

The practical implementation of the model outlined above consists of an iterative procedure searching for a steady-state solution, as shown in Fig. 2.

The stochastic aspects of charge carrier motion in disordered semiconductors are accounted for through the multiple trapping and release process of individual particles. For any process-trapping, release, recombination, photocreationoccurring at a rate $v$, the time needed for a particular instance is calculated as

$$
t_{i}=-\frac{\ln X_{i}}{v}
$$

where $X$ is a random number between 0 and 1. For example, $t_{\text {free }}$ is calculated by using the trapping rate $v_{0}$ in Eq. (10).

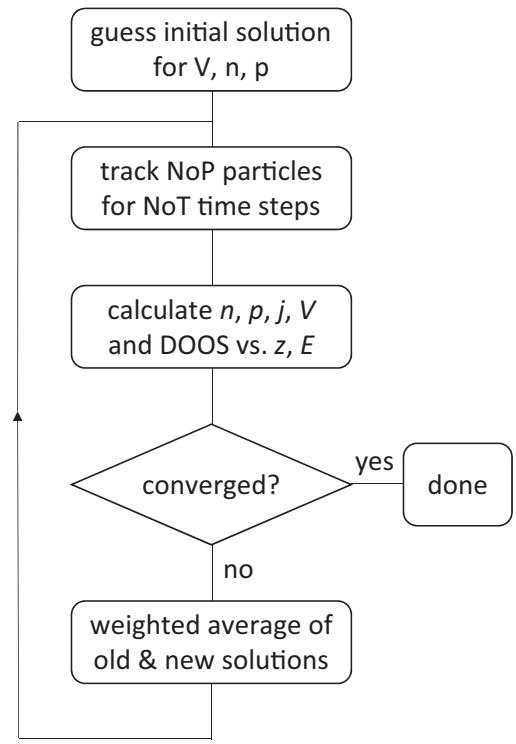

FIG. 2. Flow diagram of the numerical implementation of the $\mathrm{kDD}$ model. Typical values for both the number of particles (NoP) and the number of time steps (NoT) are $10^{3}$. When a particle annihilates by recombination or extraction at one of the contacts within NoT, it is recreated by injection or photogeneration. The weight factor for the new solution when averaging with the old one is typically $0.2-0.05$.

Recombination while the carrier is free (trapped) happens when the corresponding random recombination time is shorter than the random time spent at the transport level (trap). Likewise, trap energies are selected randomly for each trapping event from the DOS in Eq. (1), under the conditions that (i) the site is below the mobility edge at energy $E_{c}$ and (ii) the site is empty, i.e., a term $1-f(E, z)$, with $f(E, z)$, the relative occupation, is used as weight factor. This means that there is no memory of trap energies, and a particle revisiting the same position will in all likelihood get trapped at a different energy than before. It is therefore not needed to perform explicit averaging over multiple ensembles of random trap energy configurations, as is commonly done in kinetic MC calculations.

Injection is assumed to occur from the Fermi level of the metal contact $E_{F, c}$ to trap states at $z_{c}=0$ or $z_{c}=L$ at the following rate:

$$
\nu_{\text {inj }}=\frac{\nu_{0}}{\beta N_{i}} \int_{-\infty}^{E_{c}}\left(1-f\left(E, z_{c}\right)\right) g_{0}(E) \exp \left(-\frac{E-E_{F, c}}{k_{B} T}\right) d E
$$

As for trapping from the transport energy, the final site energy for a particular injection event is randomly selected using criteria (i) and (ii) above. In addition, the probability for thermal activation — second term in the integral of Eq. (11) - is used as weight factor for injection; cf. Eq. (2).

The density of occupied states $(\operatorname{DOOS})(E, z)$ is calculated on a two-dimensional grid, with $a_{\mathrm{NN}}$ as a spatial lattice constant. Along the energy dimension, typically $k_{B} T / 20$ is used as grid spacing. In particular,

$$
\operatorname{DOOS}(E, z)=\min \left(\frac{\beta N_{i}}{\sqrt{2 \pi} \sigma_{\mathrm{DOS}}} \frac{\sum \Delta t_{i}(E, z)}{t_{\mathrm{inj}}^{\mathrm{total}}}, g_{0}(E)\right)
$$


where the sum runs over all events $i$ at which a time $\Delta t_{i}$ is spent at (the grid point at) $E$ and $z$ and $t_{\text {inj }}^{\text {total }}$ is the total time associated with all injection events, i.e., the summed injection time of all charges considered in the iteration (Fig. 2). For photocreated charges, the sum of all generation times is used. The min() function assures numerical noise does not lead to unphysical occupations; i.e., for the relative occupation, $f(E, z)=\operatorname{DOOS}(E, z) / g_{0}(E) \leqslant 1$ must hold. From the DOOS, the local charge density is obtained as follows:

$$
n(z)=\int_{-\infty}^{\infty} \operatorname{DOOS}(E, z) d E
$$

In a similar fashion, the current density is calculated as

$$
j= \pm \frac{q}{a_{\mathrm{NN}}^{2}} \frac{N_{\mathrm{trans}}^{\text {total }}}{t_{\mathrm{inj}}^{\text {total }}}
$$

where the plus and minus signs are for holes and electrons, respectively and $N_{\text {trans }}^{\text {total }}$ is the total net number of transported charges in the iteration.

We noticed that significantly faster convergence can often be achieved by artificially enhancing the number of charges that are present in and pass through the device. This is done by dividing the charge density (Eq. (13)) by a factor in the range 10-20, which has a roughly proportional effect on calculation time. To compensate, the current density (Eq. (14)) and the relative occupation $f$ are divided by the same factor. The induced error in the key observables can be quite small, allowing significant speed-ups after this error has been checked. Even without this trick, the model is computationally inexpensive, making it possible to extract parameters from experiments by least-square fitting.

Although the procedure outlined above leads to steady-state solutions, it should be stressed that it is straightforward to extract transient information from the traces of the individual charges in the converged solution. Below, the energetic relaxation of photocreated charges will be compared to the experiment as a relevant example.

\section{Kinetic MC}

The kinetic MC model to which we will compare our results has been described in detail in a number of previous publications [21,22]. In brief, it accounts for nearest-neighbor hopping in a Gaussian DOS of width $\sigma_{\text {DOS }}$ according to MillerAbrahams rates with attempt frequency $v_{0}$ on a simple cubic lattice with an intersite distance $a_{\mathrm{NN}}$. Coulomb interactions between all charges and their images in the metal electrodes are exactly accounted for.

\section{EXPERIMENTS}

Bulk heterojunction hole-only devices were fabricated on precleaned Indium-tin-oxide (ITO) substrates with $40 \mathrm{~nm}$ of poly-(3,4-ethylenedioxythiophene) polystyrene sulfonate (PEDOT:PSS) spin-coated in air prior to active layer deposition. The active layer was spin-coated inside a $\mathrm{N}_{2}$-filled glovebox from a TQ1 : $\mathrm{PC}_{60} \mathrm{BM}$ solution (1:2 ratio by weight) with a concentration of $20 \mathrm{~g} \mathrm{l}^{-1}$ in 1,2-dichlorobenzene (ODCB) yielding a film thickness of 105-110 nm. The $\mathrm{MoO}_{3} / \mathrm{Al}$ top contact $(10 / 90 \mathrm{~nm})$ was evaporated on the active layer at a vacuum below $4 \times 10^{-6}$ mbar. Temperature-dependent current-voltage $(j V)$ curves on hole-only devices were measured in the dark in a high-vacuum probe station $\left(\sim 10^{-5} \mathrm{mbar}\right)$ cooled by liquid nitrogen. Fabrication of organic photovoltaic devices used in this paper is described in an earlier paper [21]

Time-resolved charge extraction measurements were performed on encapsulated organic photovoltaic devices. Briefly, we utilize the electric-field-induced second-harmonic (EFISH) generation effect to monitor the temporal evolution of the electric field strength in the active layer of the organic photovoltaic device. The motion of photocreated charges partially screens the electric field in the bulk of the active layer, thereby reducing the second-harmonic intensity and allowing for charge transport to be evaluated with sub-picosecond time resolution. The time delay between the $400 \mathrm{~nm}$ pump pulse and the $800 \mathrm{~nm}$ probe was varied by a mechanical delay stage, allowing for time delays up to $\sim 3 \mathrm{~ns}$. The later part of the transient $>3$ ns was simultaneously recorded (same pump pulse) by a transient photocurrent measurement utilizing an oscilloscope with a $10 \mathrm{k} \Omega$ load (integrated photocurrent). The outlined method and the time-resolved EFISH technique (TREFISH) in particular have been described in detail elsewhere; see Refs. [31] and [21].

\section{RESULTS}

The open symbols in Fig. 3(a) show the temperaturedependent $j V$ curves measured for hole-only TQ1:PCBM diodes. In order to not have to deal with ambiguities related to leakage and background doping, we focus our analysis on the space-charge limited regime above $\sim 1 \mathrm{~V}$. The connected solid symbols are the results from the MC model after an elaborate manual optimization of the hole transport parameters. The agreement is excellent.

In Fig. 3(b), we compare the MC calculations with the predictions of the $\mathrm{kDD}$ model using the same input parameters. Over the largest part of the parameter space, especially around room temperature, i.e., the experimentally most relevant range $\hat{\sigma}=3-4$, the correspondence is good. This implies that under the near-equilibrium conditions of space-charge limited conduction, the kDD model gives an accurate representation of the extended Gaussian disorder model (EGDM) [3]. At larger $\hat{\sigma}$, the temperature and especially the field dependence start to deviate, which we tentatively attribute to the somewhat ad hoc implementation of the Ohmic contacts and possibly the ansatz Eq. (5). However, we should stress that Eq. (5), and the particular scaling of the field-energy $q a_{\mathrm{NN}} F$ with the disorder $\sigma_{\mathrm{DOS}}$ and not the thermal energy $k_{B} T$, are essential to reach agreement with both MC and experiments at higher fields.

In order to further test the accuracy of the kDD model, we compare in Fig. 4 the $j V$ curves for various thicknesses calculated by MC and $\mathrm{kDD}$ on basis of the same parameter set used in Fig. 3. The ability to correctly capture the thickness dependence of space-charge limited currents in organic semiconductors was previously shown to be a distinctive quality of the EGDM, implying that the field and temperature dependence of charge transport have been correctly captured [6]. Indeed, the agreement between the curves in Fig. 4 is satisfactory. We attribute the minor deviations that arise at 

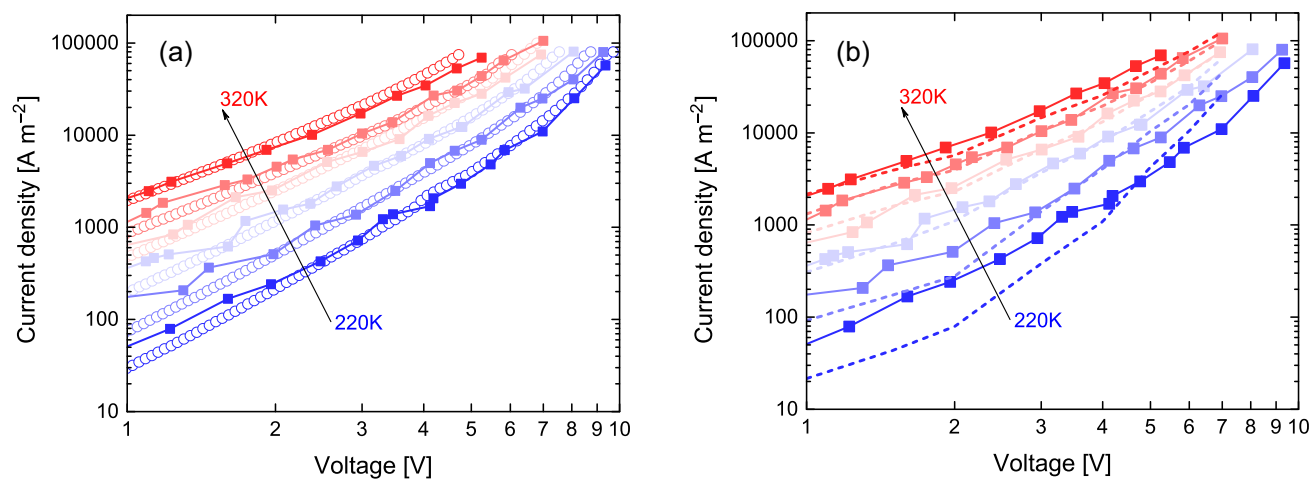

FIG. 3. (a) Temperature-dependent current-voltage characteristics of a hole-only TQ1:PCBM device with $L=110 \mathrm{~nm}$. Open symbols are experiments, and connected solid symbols are calculations by MC. (b) Comparison between calculations by MC (connected solid symbols) and $\mathrm{kDD}$ (dashed lines). Simulation parameters are $\sigma_{\mathrm{DOS}}=0.085 \mathrm{eV}, v_{0}=5.53 \times 10^{10} \mathrm{~s}^{-1}$, and $a_{\mathrm{NN}}=1.8 \mathrm{~nm}$, giving $\mu_{0}=1.8 \times 10^{-8} \mathrm{~m}^{2} \mathrm{Vs}^{-1}$. Injection barriers of $0.2 \mathrm{eV}$ were used for the Ohmic contacts.

low and intermediate field strengths for the $50 \mathrm{~nm}$ device to the $\mathrm{kDD}$ model not accurately describing the rare but highconductivity percolation pathways that dominate the transport through very thin layers $[32,33]$. For the same thickness at high voltages, i.e., very high fields, the Miller-Abrahams hopping rates used in the $\mathrm{MC}$ calculations produce a well-understood artifact in the form of a decreasing mobility when most hops are down in energy, giving rise to hopping rates and conductivities that level off. In the kDD formalism, this does not happen.

Having established good performance of our model under steady-state near-equilibrium conditions, we now turn to the limit of ultrafast charge transport of photocreated charges, where transient and far-from-equilibrium phenomena are expected to dominate. In Fig. 5(a), we compare simulated charge extraction experiments using the same hole hopping parameters as used in Figs. 3 and 4. Electron parameters were taken from Ref. [21].

We have previously shown that TREFISH plus transient photocurrent experiments directly probe the ultrafast charge carrier dynamics in disordered organic semiconductors, as well as the strong dispersion in extraction times of photocreated

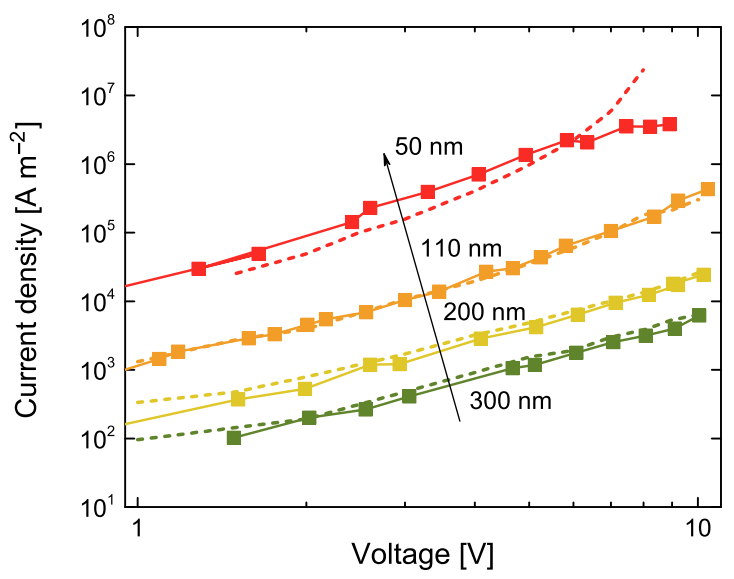

FIG. 4. Thickness dependence of space-charge limited conduction as calculated by MC (connected solid symbols) and $\mathrm{kDD}$ (dashed lines). $T=300 \mathrm{~K}$, and $L=50,110,200$, and $300 \mathrm{~nm}$; otherwise, the same parameters are used as in Fig. 3. charge carriers [21]. Moreover, it was found that these can be consistently described and understood on the basis of kinetic MC models that account for the slow energetic relaxation of charges in a Gaussian-shaped DOS [22]. The overall good agreement with MC calculations shows that the $\mathrm{kDD}$ model captures these phenomena to a large degree.

As an example of the practical potential of our model, we used it to reanalyze the previously published data set from Ref. [21], as shown in Fig. 5(b). The low computational cost of the $\mathrm{kDD}$ formalism allowed use of a least-squares fitting procedure. Compared to the previously published fit that was based on MC calculations-see the thin dashed lines in Fig. 5(b) - the agreement between experiment and model has somewhat increased. The calculation time required to do this has gone down by more than an order of magnitude. The difference in the extracted hopping parameters (e.g., for the holes, $\sigma_{\mathrm{DOS}}=0.113 \mathrm{eV}$ and $\nu_{0}=4.93 \times 10^{10} \mathrm{~s}^{-1} \mathrm{vs}$ $\sigma_{\text {DOS }}=0.10 \mathrm{eV}$ and $\nu_{0}=1 \times 10^{10} \mathrm{~s}^{-1}$ in Ref. [21]) reflects the interchangeability of disorder and attempt frequency that we signaled in our earlier paper. Therefore, within the limits of the Gaussian disorder model, using kDD and MC to analyze the same experimental data set leads to extracted hopping parameters that are largely equivalent—using Eqs. (3) and (4), these parameters correspond to steady-state hole mobilities of $\mu_{0}=$ $3.2 \times 10^{-10} \mathrm{~m}^{2} \mathrm{Vs}^{-1}$ and $\mu_{0}=4.5 \times 10^{-10} \mathrm{~m}^{2} \mathrm{Vs}^{-1}$, respectively, in good agreement with the experimental photo-charge extraction by linearly increasing voltage (photo-CELIV) mobility of pristine TQ1 films $\mu=6 \times 10^{-10} \mathrm{~m}^{2} \mathrm{Vs}^{-1}$ [21].

Deviations from experiment are mainly present in the first part of the charge extraction transient, corresponding to electron extraction, especially $r$, the lowest field curve $(-1 \mathrm{~V})$, and occur for both $\mathrm{kDD}$ and MC simulations. As such, their origin must be sought in the common underlying Gaussian disorder formalism. We speculate that the electron DOS might deviate from a strict Gaussian. We attribute the fact that different parameters are needed to describe the hole transport in Figs. 3 and 5 to the fact that the shown experiments have been performed on different samples made from different batches of the TQ1 polymer. Moreover, we suspect some deterioration of the devices under the time-consuming TREFISH plus transient photocurrent measurements, possibly leading to a somewhat 

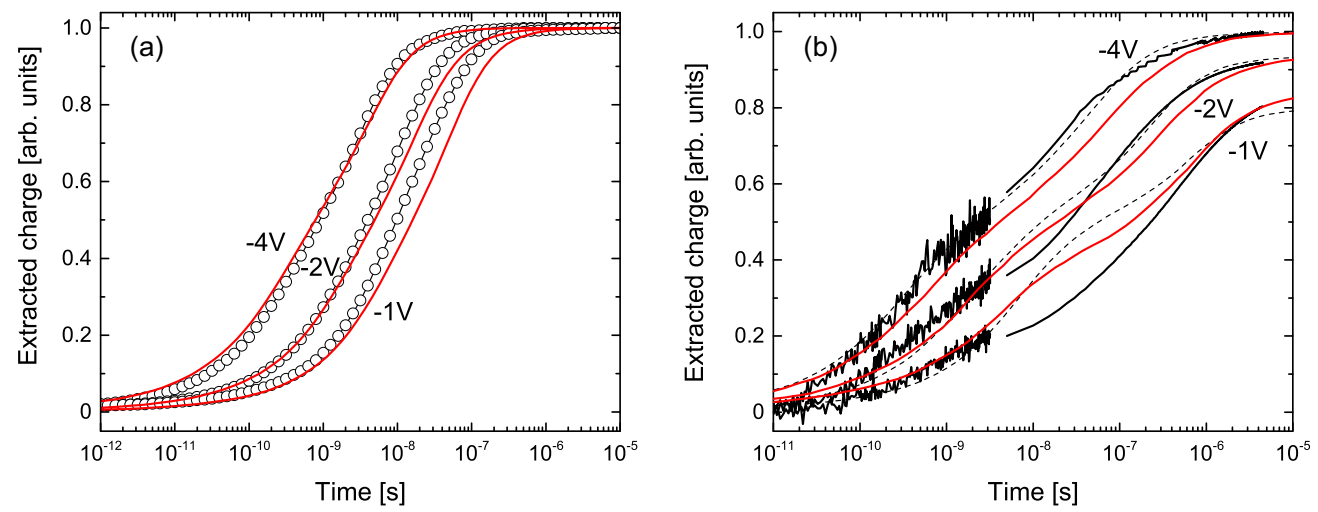

FIG. 5. Simulation of combined TREFISH and transient photocurrent experiments. (a) Extracted charge vs time as calculated by MC (connected open symbols) and $\mathrm{kDD}$ (solid red lines), using the hole parameters from Fig. 3 and electron parameters $\sigma_{\mathrm{DOS}}=0.12 \mathrm{eV}$, $v_{0}=1 \times 10^{13} \mathrm{~s}^{-1}, L=70 \mathrm{~nm}$, and $T=300 \mathrm{~K}$ from Ref. [21]. (b) Measured (heavy black lines; data taken from Ref. [21]) and calculated by $\mathrm{kDD}$ (solid red lines) charge extraction for a TQ1 : $\mathrm{PC}_{71} \mathrm{BM}$ organic photovoltaic device. Parameters are obtained by a least-squares fitting procedure and are $\sigma_{\text {DOS }}=0.125 \mathrm{eV}$ and $\nu_{0}=1.84 \times 10^{13} \mathrm{~s}^{-1}$ for electrons and $\sigma_{\text {DOS }}=0.113 \mathrm{eV}$ and $\nu_{0}=4.93 \times 10^{10} \mathrm{~s}^{-1}$ for holes. The thin dashed lines are an MC calculation using the parameters from Ref. [21]: $\sigma_{\text {DOS }}=0.10 \mathrm{eV}$ and $\nu_{0}=1 \times 10^{10} \mathrm{~s}^{-1}$ for holes and $\sigma_{\text {DOS }}=0.12 \mathrm{eV}$ and $v_{0}=1 \times 10^{13} \mathrm{~s}^{-1}$ for electrons.

increased hole disorder and concomitantly a reduced hole mobility.

The dispersive transport that is probed in the combined TREFISH and transient photocurrent experiment of Fig. 5 is driven by the slow relaxation process of photocreated charge carriers [21,22,34]. In Fig. 6, we plotted the calculated energy loss with respect to the center of the respective (electron or hole) DOS. The correspondence between MC and kDD models is perfect - the difference at very short timescales is due to the trivial fact that the MC and kDD models initialize the charge carrier distributions differently. MC selects random energies in the DOS (Eq. (1)), so on average, charges are generated in the DOS center, leading to zero loss at $t=0$, whereas $\mathrm{kDD}$ initializes charge carriers at the transport energy (Eq. (5)). The curves in Fig. 6 are not strictly log-linear [1], as would be the case for an exponential DOS [18,19]. Nevertheless, the physical reason for the slowing of the relaxation process is the same: the further charges relax, the longer they will be stuck in their current site and the rarer even deeper sites become.

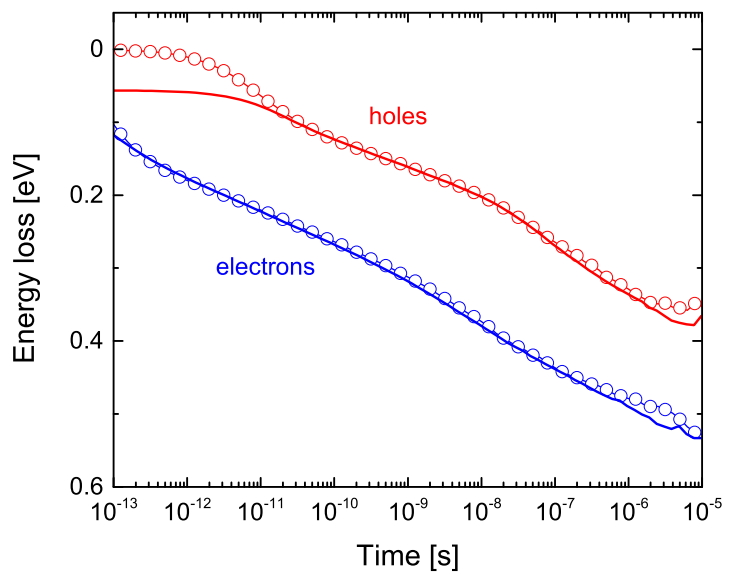

FIG. 6. Time-dependent relaxation of photocreated electrons and holes as calculated by MC (symbols) and kDD (lines). Same parameters as Fig. 5(a).
Finally, we should point out that in the time interval relevant for the extraction of photocreated charges, cf. Fig. 5, neither the electrons nor the holes fully reach their equilibrium energy $E_{\infty}=\sigma^{2} / k_{B} T$ of $\sim 0.56 \mathrm{eV}$ for electrons and $\sim 0.39 \mathrm{eV}$ for holes, in agreement with experiments [22].

\section{CONCLUSION}

We have developed a nonequilibrium DD model that naturally takes into account the characteristic aspects of charge carrier motion in disordered organic semiconductor materials: a density and electric field-dependent mobility under steadystate conditions and highly dispersive and fast charge transport when the charge population has not yet reached thermal equilibrium. The latter is typically the case for photocreated charges. We benchmarked our kDD model to the more timeconsuming kinetic MC calculations and used it to successfully describe both direct current and ultrafast charge transport experiments. In addition, the slow relaxation process of nonequilibrium charge carriers in the DOS is accurately described.

\section{APPENDIX: DIVERGENCE OF IMAGE POTENTIAL}

The divergence of the image potential in Eq. (8) at $z=0$ and $z=L$ is numerically troublesome. When correct treatment of the image potential is imperative, as is the case in Figs. 3 and 4 , an approximate value for $V_{\text {im }}$ is used at the contacts. In particular, we used

$$
V_{\mathrm{im}}(0)=V_{\mathrm{im}}\left(a_{\mathrm{NN}}\right)-Z_{1}\left(V_{\mathrm{im}}\left(2 a_{\mathrm{NN}}\right)-V_{\mathrm{im}}\left(a_{\mathrm{NN}}\right)\right)
$$

with the empirical prefactor $Z_{1}>0$. A similar expression for the contact at $z=L$ is used. For $Z_{1}$ substantially larger than unity, it becomes virtually impossible for charges to diffuse into the device - the image potential leads to a field that is directed to the contact, precluding drift motion away from the contact. We solved this by artificially increasing the diffusion constant (Eq. (6)) at $z=0$ and $z=L$ by a factor $Z_{2}>1$. The exact outcome of the model calculations is fortunately only a weak function of $Z_{1}, Z_{2}$, provided both are sufficiently large. 
Moreover, only the low-field regime is affected by the choice of $Z_{1}, Z_{2}$. Unfortunately, calculation times increase drastically for large $Z_{1}$. We used $Z_{1}=Z_{2}=10$ as a good compromise when fitting the hole-only diode experiments in Figs. 3 and 4 and thus recommend the use of these numbers when fitting space-charge limited currents experiments. For simulations of TREFISH + transient photocurrent, which are inherently less dependent on the choice of contacts, we suggest the use of the (much) faster combination $Z_{1}=3, Z_{2}=1$, which was used in this paper.
[1] H. Bässler, Phys. Status Solidi B 175, 15 (1993).

[2] W. F. Pasveer, J. Cottaar, C. Tanase, R. Coehoorn, P. A. Bobbert, P. W. M. Blom, D. M. de Leeuw, and M. A. J. Michels, Phys. Rev. Lett. 94, 206601 (2005).

[3] R. Coehoorn, W. F. Pasveer, P. A. Bobbert, and M. A. J. Michels, Phys. Rev. B 72, 155206 (2005).

[4] H. Bässler and A. Köhler, in Unimolecular and Supramolecular Electronics I, edited by R. M. Metzger (Springer, Berlin, 2011), p. 65.

[5] S. D. Baranovskii, Phys. Status Solidi B 251, 487 (2014).

[6] P. W. M. Blom, C. Tanase, D. M. de Leeuw, and R. Coehoorn, Appl. Phys. Lett. 86, 092105 (2005).

[7] S. L. M. van Mensfoort and R. Coehoorn, Phys. Rev. B 78, 085207 (2008).

[8] R. J. de Vries, S. L. M. van Mensfoort, V. Shabro, S. I. E. Vulto, R. A. J. Janssen, and R. Coehoorn, Appl. Phys. Lett. 94, 163307 (2009).

[9] M. Kuik, G.-J. A. H. Wetzelaer, H. T. Nicolai, N. I. Craciun, D. M. De Leeuw, and P. W. M. Blom, Adv. Mater. 26, 512 (2014).

[10] S. V. Novikov, D. H. Dunlap, V. M. Kenkre, P. E. Parris, and A. V. Vannikov, Phys. Rev. Lett. 81, 4472 (1998).

[11] M. Bouhassoune, S. L. M. van Mensfoort, P. A. Bobbert, and R. Coehoorn, Org. Electron. 10, 437 (2009).

[12] M. Vissenberg and M. Matters, Phys. Rev. B 57, 12964 (1998).

[13] A. V. Nenashev, F. Jansson, J. O. Oelerich, D. Huemmer, A. V. Dvurechenskii, F. Gebhard, and S. D. Baranovskii, Phys. Rev. B 87, 235204 (2013).

[14] J. O. Oelerich, D. Huemmer, and S. D. Baranovskii, Phys. Rev. Lett. 108, 226403 (2012).

[15] I. Hwang, C. R. McNeill, and N. C. Greenham, J. Appl. Phys. 106, 094506 (2009).
[16] T. Kirchartz and J. Nelson, Phys. Rev. B 86, 165201 (2012).

[17] T. Kirchartz and J. Nelson, Top. Curr. Chem. 352, 279 (2013).

[18] H. Scher and E. W. Montroll, Phys. Rev. B 12, 2455 (1975).

[19] T. Tiedje and A. Rose, Solid State Comm. 37, 49 (1981).

[20] M. Silver, L. Pautmeier, and H. Bässler, Solid State Comm. 72, 177 (1989).

[21] A. Melianas, V. Pranculis, A. Devižis, V. Gulbinas, O. Inganäs, and M. Kemerink, Adv. Funct. Mater. 24, 4507 (2014).

[22] A. Melianas, F. Etzold, T. J. Savenije, F. Laquai, O. Inganäs, and M. Kemerink, Nat. Comm. 6, 8778 (2015).

[23] J. Noolandi, Phys. Rev. B 16, 4474 (1977).

[24] J. Orenstein and M. A. Kastner, Solid State Comm. 40, 85 (1981).

[25] D. Monroe, Phys. Rev. Lett. 54, 146 (1985).

[26] W. C. Germs, J. J. M. van der Holst, S. L. M. van Mensfoort, P. A. Bobbert, and R. Coehoorn, Phys. Rev. B 84, 165210 (2011).

[27] J. Cottaar, L. J. A. Koster, R. Coehoorn, and P. A. Bobbert, Phys. Rev. Lett. 107, 136601 (2011).

[28] A. Salleo, T. W. Chen, A. R. Völkel, Y. Wu, P. Liu, B. S. Ong, and R. A. Street, Phys. Rev. B 70, 115311 (2004).

[29] J. G. Simmons, J. Appl. Phys. 34, 1793 (1963).

[30] M. Kuik, L. J. A. Koster, G. A. H. Wetzelaer, and P. W. M. Blom, Phys. Rev. Lett. 107, 256805 (2011).

[31] A. Devižis, A. Serbenta, K. Meerholz, D. Hertel, and V. Gulbinas, Phys. Rev. Lett. 103, 027404 (2009).

[32] B. I. Shklovskii and A. L. Efros, Electronic Properties of Doped Semiconductors (Springer-Verlag, Heidelberg, 1984).

[33] M. Pollak and J. J. Hauser, Phys. Rev. Lett. 31, 1304 (1973).

[34] I. A. Howard, F. Etzold, F. Laquai, and M. Kemerink, Adv. Energy Mater. 4, 1301743 (2014). 